\title{
ARTICLE
}

Received 10 Apr 2013 | Accepted 26 Sep 2013 | Published 25 Oct $2013 \quad$ DOl: 10.1038/ncomms3672

\section{Semiconductor-based DNA sequencing of histone modification states}

Christine S. Cheng ${ }^{1}$, Kunal Rai ${ }^{2}$, Manuel Garber ${ }^{1,3}$, Andrew Hollinger ${ }^{1}$, Dana Robbins ${ }^{1}$, Scott Anderson ${ }^{1}$, Alyssa Macbeth ${ }^{1}$, Austin Tzou1, Mauricio O. Carneiro ${ }^{1}$, Raktima Raychowdhury ${ }^{1}$, Carsten Russ ${ }^{1}$, Nir Hacohen 1,4,5, Jeffrey E. Gershenwald ${ }^{6}$, Niall Lennon ${ }^{1}$, Chad Nusbaum¹, Lynda Chin ${ }^{2}$, Aviv Regev ${ }^{1,7}$ \& Ido Amit ${ }^{1,8}$

The recent development of a semiconductor-based, non-optical DNA sequencing technology promises scalable, low-cost and rapid sequence data production. The technology has previously been applied mainly to genomic sequencing and targeted re-sequencing. Here we demonstrate the utility of Ion Torrent semiconductor-based sequencing for sensitive, efficient and rapid chromatin immunoprecipitation followed by sequencing (ChIP-seq) through the application of sample preparation methods that are optimized for ChIP-seq on the lon Torrent platform. We leverage this method for epigenetic profiling of tumour tissues.

\footnotetext{
${ }^{1}$ Broad Institute of MIT and Harvard, Cambridge, Massachusetts 02142, USA. ${ }^{2}$ Department of Genomic Medicine, University of Texas MD Anderson Cancer Center, Houston, Texas 77030, USA. ${ }^{3}$ Bioinformatics and Integrative Biology, University of Massachusetts Medical School, Worcester, Massachusetts 01605 , USA. ${ }^{4}$ Center for Immunology and Inflammatory Diseases, Massachusetts General Hospital, Charlestown, Massachusetts 02129, USA. ${ }^{5}$ Department of Medicine, Harvard Medical School, Boston, Massachusetts 02115, USA. ${ }^{6}$ Department of Surgical Oncology, University of Texas MD Anderson Cancer Center, Houston, Texas 77030, USA. 7 Howard Hughes Medical Institute, Department of Biology, Massachusetts Institute of Technology, Cambridge, Massachusetts 02142, USA. ${ }^{8}$ Department of Immunology, Weizmann Institute, Rehovot 76100, Israel. Correspondence and requests for materials should be addressed to L.C (email: Ichin@mdanderson.org) or to A.R. (email: aregev@broadinstitute.org) or to I.A. (email: ido.amit@weizmann.ac.il).
} 
T he semiconductor-based, non-optical sequencing technology used by the Ion Torrent sequencer (Life Technologies, Carlsbad, CA) has the potential for scalable, rapid and lowcost sequence data production ${ }^{1}$. Given the current industry standard for the density of transistors on the surface of a semiconductor, the technology has not yet reached its full possible capacity ${ }^{2}$ and has the potential to provide comparable sequencing data yields to conventional optical-based sequencers in a fraction of the time and $\cos ^{3,4}$. The technology has recently been applied to genomic sequencing ${ }^{1}$, microbial genotyping ${ }^{5}$ and targeted re-sequencing ${ }^{6}$.

Chromatin immunoprecipitation followed by high-throughput sequencing (ChIP-seq) is a powerful tool for characterizing the epigenetic landscape and transcriptional network in the context of both normal physiology and disease ${ }^{7-9}$. However, Ion Torrent sequencing has not yet been used for ChIP-Seq because of challenges in using ChIP DNA samples for sequencing library preparation. First, ChIP yields relatively low amounts of DNA, whereas commercial ChIP-Seq protocols recommend at least $500 \mathrm{ng}$ to $1 \mu \mathrm{g}$ of starting material for the library construction process. This is an issue in particular for ChIP DNA samples from immunoprecipitation of transcription factors or from limiting samples such as rare cell types or clinical samples, which are often at the few nanograms range. Although recent studies presented ChIP-Seq protocols with low input (low cell number) for the Illumina platform ${ }^{10,11}$, such protocols are not yet available for the Ion Torrent platform. Second, the Ion Torrent process works optimally with a tight size range of DNA molecules of $\sim 280 \pm 20 \mathrm{bp}$, whereas ChIP DNA typically spans a range of sizes from 200 to $600 \mathrm{bp}$.

Here we demonstrate the utility of Ion Torrent sequencing for ChIP-seq samples with sub-nanogram amounts of DNA. Further, we apply the method to profile epigenetic marks of tumour tissues from melanoma patients and show its potential for analyzing tumour progression.

\section{Results}

Development of a ChIP-seq application for Ion Torrent. Our starting point was an automated 454-library construction method we previously developed ${ }^{12}$. To overcome the low-input material obtained by ChIP, we devised a low input, scalable and robust library construction protocol for ChIP DNA that increases sensitivity and minimizes operator-dependent variability by incorporating a high yielding amplification enzyme (Kapa Biosystems, Woburn, MA), which has higher yield and higher genome coverage $^{13}$ than the Phusion polymerase that is commonly used in the standard Illumina protocol, low microlitre volume reactions, molecularly barcoded oligonucleotide adaptors and automated fluid-handling protocols (Supplementary Fig. S1 and Methods)

To address the wide size range of ChIP DNA, we first tested a standard enzymatic DNA-shearing method that is routinely used with Ion Torrent genomic libraries, but failed to generate usable ChIP-Seq libraries. To overcome this problem, we started the library construction process without shearing and then used an automated gel size-selection system (Pippin Prep, Sage Science, Beverly, MA) to select appropriately sized library molecules after adaptor ligation. We note that Illumina ChIP-seq libraries are usually not sheared, as the sheering step results in significant material loss, which is of particular concern with very low-input samples, such as ChIP samples. Using this process, we successfully created libraries for 32 of 36 samples attempted (88.9\% pass rate; success defined as having sufficient library material to attempt at least three sequencing reactions. An Illumina ChIP-Seq library construction following a successful ChIP is closer to $100 \%$ ).
To compare results between Ion Torrent sequencing and those from Illumina sequencing for ChIP applications, we performed ChIP with antibodies to the common histone mark, histone 3 lysine 4 tri-methyl (H3K4me3), the carboxy-terminal domain of RNA polymerase II (Pol-II) and IgG (negative control) in mouse dendritic cells stimulated with lipopolysaccharide. The resulting immunoprecipitated DNA was used as an input for both our Ion Torrent and standard Illumina library construction procedures. We sequenced the libraries on the Ion Torrent 316 sequencing chips (on average, two million reads per library, average read length: 180 bases) and with the gold standard ChIP-Seq data production using the Illumina HiSeq 2000 (15 million reads per library; read length: 40 base single end $)^{7,14,15}$.

Illumina has a lower percentage of unmapped bases and a significantly higher rate of well-mapped bases than Ion Torrent (Supplementary Table S1). Although the Ion Torrent reads had higher error rates for both SNPs (10-fold higher) and indels (100fold higher), these were still below 1 in 1,000 bases (Supplementary Table S1) and thus do not have an impact on the quality of the chromatin maps.

We found excellent agreement between the two resulting maps. The ChIP-Seq enrichment scores, defined as the ratio of observed/expected number of reads at each peak region, are highly correlated between the two samples (H3K4me3: Pearson's $R=0.893$, Pol-II: $R=0.722$, Fig. 1a,b). Despite the differences in mapped bases and error rates, Ion Torrent sequencing produced comparable enrichment peaks to Illumina ${ }^{5,9,10}$ sequencing for both H3K4me3 and Pol-II ChIP-seq (Fig. 1a,b and Supplementary Fig. S2). Saturation analysis by subsampling the Ion Torrent reads indicates that the Ion Torrent library was sequenced to sufficient depth at two million reads (Fig. 1c). To examine the possibility that the longer read length in Ion Torrent sequencing reads contributes to this phenomenon, we extended the 40 base reads from Illumina to 180 bases. The extended Illumina reads (randomly down sampled from 15 million to 2 million reads) produced enrichment peaks that were more comparable to the two million Ion Torrent reads, indicating that longer read lengths may be beneficial in ChIP-seq applications independent of the sequencing platform used (Supplementary Fig. S3). Similarly, when we shortened the Ion Torrent reads from 180 bases to 40 bases and then performed alignment, the enrichment peaks were reduced, similar to Illumina reads down sampled to two million reads (40 bases; Supplementary Fig. S3). As expected, we did not find enriched peaks with a negative control IgG ChIP-seq from Ion Torrent sequencing (Supplementary Fig. S4).

To determine the sensitivity of Ion Torrent sequencing for ChIP-seq, we tested a titration of ChIP DNA input amounts. We used 56, 4 and $0.4 \mathrm{ng}$ of H3K4me3 ChIP DNA from a single ChIP experiment as input for our modified library construction protocol for Ion Torrent. We obtained successful libraries from all three aliquots with comparable results (Fig. 1d-f). For example, the lowest input library $(0.4 \mathrm{ng}$; equivalent to H3K4me3-immunoprecipitated DNA from 20,000 cells) was comparable to the highest input library (56 ng; equivalent to $10 \times 10^{6}$ cells) based on enriched peaks (Fig. 1c) and correlation in enriched scores $(R=0.753$, Fig. 1f). Such low ChIP DNA input amounts are an order of magnitude lower than the Ion Torrent guidelines for library production and sequencing, and are comparable to recently developed protocols ${ }^{10,11}$ for low input ChIP-seq with the Illumina platform. We also tested $0.05 \mathrm{ng}$ of ChIP DNA, but failed to produce a high-quality library. In summary, using our protocol we created successful libraries from very low starting amounts of ChIP DNA and obtained comparable results with Ion Torrent ChIP-seq to those with our standard methods, optical-based Illumina sequencing, while 
a

H3K4me3

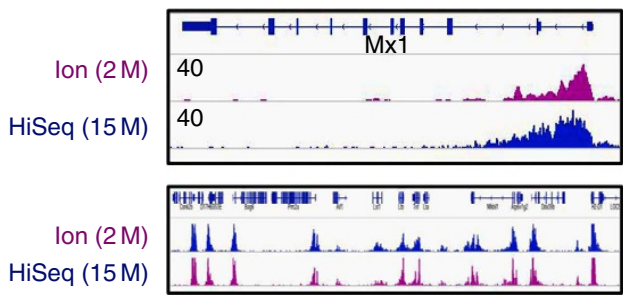

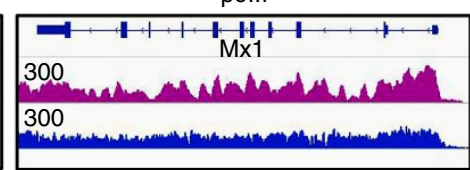

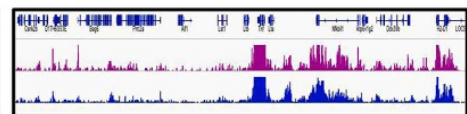

b
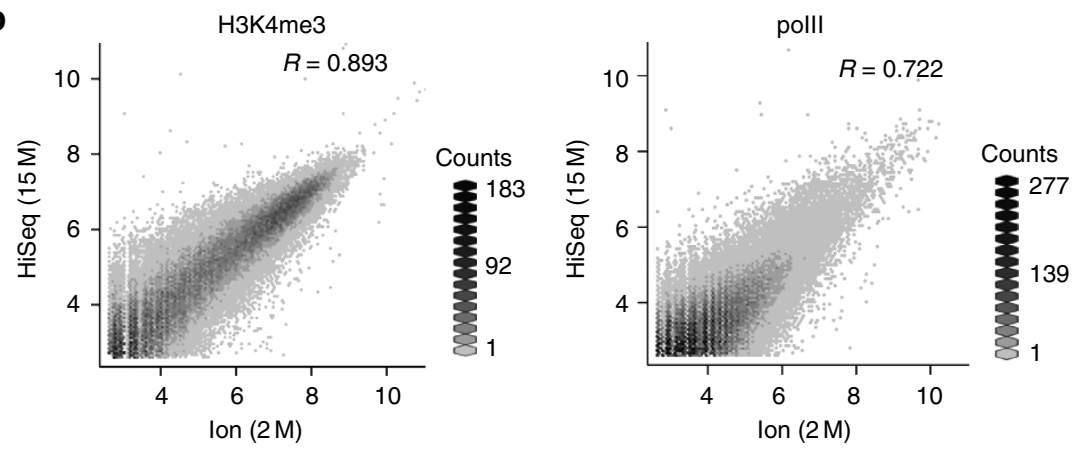

C

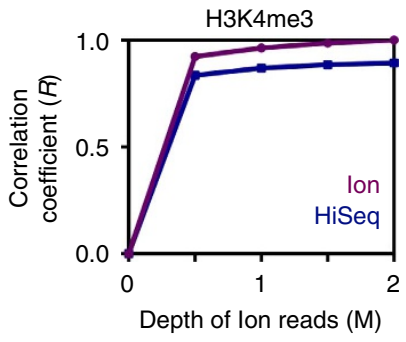

d

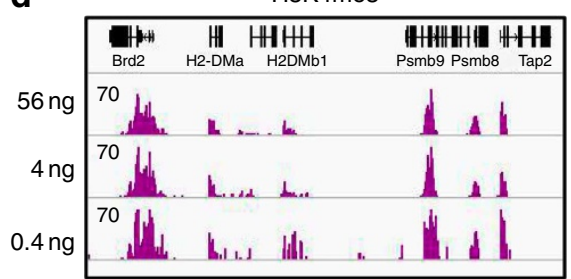

e
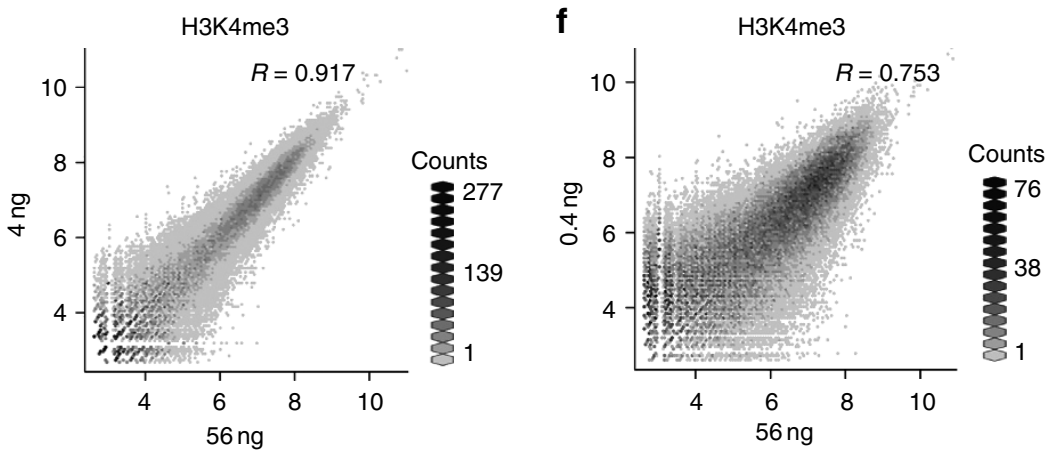

Figure 1 | Ion Torrent and Illumina HiSeq ChIP-seq. (a) Density profiles of H3K4me3 (left) and Pol-II ChIP-seq (right) from 2 million lon Torrent reads (purple) and 15 million Illumina HiSeq reads (blue) over the Mx1 gene locus (top) and a larger region spanning the TNF locus (bottom). The scale of the density profile was set to the same level for each pair of samples and is marked. All ChIP-seq experiments were performed with mouse dendritic cells stimulated with lipopolysaccharide $\left(0.1 \mu \mathrm{g} \mathrm{ml}^{-1}\right)$ for $2 \mathrm{~h}$. (b) A comparison of H3K4me3 (left) and Pol-Il (right) ChIP-seq peak enrichment scores $\left(\log _{2}\right)$ over a 500-bp sliding window from 2 million lon Torrent reads ( $x$ axis) and 15 million Illumina HiSeq reads ( $y$ axis). The Pearson correlation coefficient is marked at the upper right corner. The grey scale represents a two-dimensional density plot, where the grid is divided into hexagonal bins and the colour intensity reflects the density (counts) in the bin. An intensity bar of the counts is shown at the right bottom corner. (c) Saturation analysis to test for sufficient sequencing depth. Shown is the Pearson correlation coefficient ( $y$ axis) calculated between decreasing sequencing reads randomly subsampled from the H3K4me3 ChIP-seq lon Torrent reads ( $x$ axis) and either 15 million H3K4me3 ChIP-seq Illumina HiSeq reads (blue) or 2 million lon Torrent reads (purple). (d) Density profiles of H3K4me3 ChIP-seq from 2 million lon Torrent reads for libraries with 56, 4 or 0.4 ng of immunoprecipitated input DNA. (e,f) A comparison of H3K4me3 ChIP-seq peak enrichment scores ( $\log _{2}$ ) over a 500-bp window from 2 million lon Torrent reads with $56 \mathrm{ng}$ ( $y$ axis) and $4 \mathrm{ng}$ (d, $x$ axis) or $0.4 \mathrm{ng}$ (e, $x$ axis) of immunoprecipitated input DNA. The Pearson correlation coefficient is marked at the upper right corner. The grey scale representation as in panel $\mathbf{b}$.

requiring an order of magnitude fewer sequencing reads (albeit longer reads).

Epigenetic signatures during cancer progression. The rapid nature and relatively low cost of Ion Torrent sequencing make it a promising candidate for diagnostic applications. As a proof of principal, we next performed H3K4me3 ChIP-seq experiments with a matched pair of primary tumour and metastasis cell lines derived from the same melanoma patient. Although the matched pair WM115 (primary tumour) and WM266-4 (metastasis) showed global correlated enrichment of $\mathrm{H} 3 \mathrm{~K} 4 \mathrm{me} 3$ in gene 
promoters $\left(r^{2} R=0.83\right.$, Fig. 2a and Supplementary Fig. S5), a large number of the genes demonstrated increased levels of $\mathrm{H} 3 \mathrm{~K} 4 \mathrm{me} 3$ on their promoters in either the primary tumour (Fig. 2a,b) or metastasis (Fig. 2a,c). To test whether any biological functions were associated with differential levels of H3K4me3, we performed gene set enrichment analysis (GSEA) with the gene sets in the Molecular Signatures Database (MSigDB) ${ }^{16}$.

Interestingly, we found that decreased $\mathrm{H} 3 \mathrm{~K} 4 \mathrm{me} 3$ levels in metastasis are significantly associated with genes whose expression is repressed in embryonic stem cells, including targets of the polycomb repressive complex and for genes enriched for the H3K27me3 histone mark in embryonic stem cells ${ }^{17}$ (Fig. 2d). Conversely, increased $\mathrm{H} 3 \mathrm{~K} 4 \mathrm{me} 3$ levels in metastasis are significantly associated with interferon response and inflammatory response genes (Fig. 2e).

$\mathrm{H} 3 \mathrm{~K} 4 \mathrm{me} 3$ is mostly enriched in promoters of actively transcribed genes, whereas $\mathrm{H} 3 \mathrm{~K} 27 \mathrm{me} 3$ is usually enriched at repressive chromatin regions. In embryonic stem cells, however, many of the polycomb targets, and especially key developmental regulators, are marked by both marks ('bivalent domains') ${ }^{18}$. To explore the relationship between these two marks and metastasis, we performed $\mathrm{H} 3 \mathrm{~K} 27 \mathrm{me} 3 \mathrm{ChIP}$-seq experiments in the same cell lines. Indeed, increased $\mathrm{H} 3 \mathrm{~K} 27 \mathrm{me} 3$ levels in metastasis correlate with loss of $\mathrm{H} 3 \mathrm{~K} 4 \mathrm{me} 3$ and such genes are enriched for polycomb target genes in embryonic stem cells (Supplementary Fig. S6a). Interestingly, genes that have decreased H3K27me3 levels in metastasis are enriched for interferon response genes (Supplementary Fig. S6b; a few of the interferon response gene sets are enriched in the top 150 gene sets).

To gain initial insight into the clinical applicability of the technology, we performed H3K4me3 ChIP-seq of seven metastatic tumour tissues from melanoma patients. Consistent with our observation in the progressive cell lines, genes that have increased $\mathrm{H} 3 \mathrm{~K} 4 \mathrm{me} 3$ levels in the metastatic tumours are enriched for interferon, inflammatory and immune response genes and genes that have decreased $\mathrm{H} 3 \mathrm{~K} 4 \mathrm{me} 3$ levels are enriched for H3K27me3/polycomb target genes (Fig. 2f,g and Supplementary Figs S7-S10).

\section{Discussion}

The repression of developmental gene sets in melanoma metastasis by an $\mathrm{H} 3 \mathrm{~K} 27 \mathrm{me} 3$ gain and $\mathrm{H} 3 \mathrm{~K} 4 \mathrm{me} 3$ loss is consistent with recent findings of similar embryonic stem cell signatures in aggressive tumours from other cancers ${ }^{19,20}$. Further, several reports show that several histone-modifying enzymes are misregulated in human cancers ${ }^{21}$. For example, EZH2, an $\mathrm{H} 3 \mathrm{~K} 27 \mathrm{me} 3$ writer, is overexpressed in various solid tumours and its expression is correlated with tumour aggressiveness and metastatic progression ${ }^{22,23}$. Consistent with this, a stem cell polycomb repression signature is also enriched in genes that gain
H3K27me3 marks in metastatic prostate cancer ${ }^{24}$. Our finding that interferon and inflammatory response genes have higher levels of the H3K4me3 mark in melanoma metastasis is consistent with recent findings that link inflammation with cancer ${ }^{25-27}$.

In summary, we have demonstrated a rapid, sensitive, scalable and cost-effective semiconductor-based ChIP-seq pipeline for characterizing epigenetic signatures of metastatic human tumours from limiting samples with comparable sensitivity to recently developed protocols ${ }^{10,11}$ for ChIP-seq with low input using the Illumina platform. The technical and analytical methods for ChIP followed by Ion Torrent sequencing provide a platform for discovery and future diagnostic applications.

\section{Methods}

Cell culture and human tissues. Mouse dendritic cells were isolated from wildtype female 6-8-week old C57BL/6 mice obtained from the Jackson Laboratories and cultured in RPMI medium (Invitrogen) supplemented with $10 \%$ heat-inactivated fetal bovine serum (Invitrogen) and granulocyte macrophage-colony-stimulating factor $\left(20 \mathrm{ng} \mathrm{ml}^{-1} \text {; Peprotech, Rocky Hill, NJ }\right)^{28}$. Cells were cultured for 9 days and stimulated for $2 \mathrm{~h}$ with lipopolysaccharide $\left(100 \mathrm{ng} \mathrm{ml}^{-1}\right.$, rough, ultrapure Escherichia coli K12 strain). Paired primary melanoma tumour-derived cell line (WM115) and metastatic melanoma tumour-derived cell line (WM266-4) from the same patient were obtained from the Wistar Institute (Philadelphia, PA). Metastatic melanoma tissues were collected from the Department of Surgical Oncology, University of Texas, MD Anderson Cancer Center with informed consent of the patients and prior MIT Committee On the Use of Humans as Experimental Subjects approval.

ChIP assay. ChIP assays were performed using a previously published protocol with some minor modifications ${ }^{28}$. Briefly, cells were fixed for $10 \mathrm{~min}$ with $1 \%$ formaldehyde and quenched with glycine. Cells were lysed for $10 \mathrm{~min}$ on ice with RIPA lysis buffer (10 mM Tris- $\mathrm{HCl}$ pH 8.0, $1 \mathrm{mM}$ EDTA pH 8.0, $140 \mathrm{mM} \mathrm{NaCl}$, $1 \%$ Triton X-100, $0.1 \%$ SDS, $0.1 \%$; sodium deoxycholate for mouse dendritic cells) or RIPA lysis buffer with $0.2 \%$ SDS and without Triton X-100 (for WM115 and WM266-4 melanoma cell lines) and then sonicated using the Branson sonicator as described in Garber et al. ${ }^{28}$ Frozen human melanoma tissues $(50-100 \mathrm{mg}$ ) were thawed out on ice and chopped finely with a razor blade. Then tissues were fixed for $10 \mathrm{~min}$ with $1 \%$ formaldehyde in PBS buffer and quenched with glycine. Fixed tissues were pulverized with Covaris CryoPrep CP02 at setting 5 for two times in TT1ET tissue tube. Cells were lysed for $10 \mathrm{~min}$ on ice with $1 \%$ SDS lysis buffer (1\% SDS, $10 \mathrm{mM}$ EDTA, $50 \mathrm{mM}$ Tris- $\mathrm{HCl}, \mathrm{pH} 8.1$ ) and then sonicated using the Branson sonicator. Immunoprecipitation was performed by incubation of the sonicated cell lysate with $75 \mu \mathrm{l}$ of protein $\mathrm{G}$ magnetic dynabeads (Invitrogen) coupled to target antibody for overnight at $4^{\circ} \mathrm{C}$. Magnetic beads were then washed five times with cold RIPA buffer (10 mM Tris-HCl, pH 8.0, 1 mM EDTA, pH 8.0, $140 \mathrm{mM} \mathrm{NaCl}, 1 \%$ Triton X-100, 0.1\% SDS), twice with high-salt RIPA buffer (10 mM Tris- $\mathrm{HCl}$, pH 8.0, 1 mM EDTA, pH 8.0, $500 \mathrm{mM} \mathrm{NaCl}, 1 \%$ Triton X-100, $0.1 \%$ SDS), twice with $\mathrm{LiCl}$ buffer (10 mM Tris-HCl, pH 8.0, 1 mM EDTA, pH 8.0, $250 \mathrm{mM} \mathrm{LiCl}, 0.5 \% \mathrm{NP}-40,0.5 \%$ sodium deoxycholate), twice with TE buffer, and then eluted in $50 \mu \mathrm{l}$ of elution buffer $(10 \mathrm{mM}$ Tris-HCl, $\mathrm{pH} 8.0,5 \mathrm{mM}$ EDTA, pH $8.0,300 \mathrm{mM} \mathrm{NaCl}$ and $0.5 \% \mathrm{SDS}$ ). The eluate was reverse crosslinked at $65^{\circ} \mathrm{C}$ for $6 \mathrm{~h}$ and then treated with $2 \mu \mathrm{l}$ of RNase A (Roche Applied Science) for $30 \mathrm{~min}$ and $2.5 \mu \mathrm{l}$ of proteinase $\mathrm{K}$ (Invitrogen) for $2 \mathrm{~h}$. Finally, the de-crosslinked DNA samples were cleaned up with $120 \mu \mathrm{l}$ of solid-phase reversible immobilization (SPRI) beads and eluted in $50 \mu \mathrm{l}$ of EB buffer ( $10 \mathrm{mM}$ Tris- $\mathrm{HCl}, \mathrm{pH} 8.0)$.

Ion Torrent library production and sequencing. ChIP-Seq libraries for Ion Torrent sequencing were created using a modified protocol of the manufacturer's

Figure 2 | Epigenetic signatures correspond to cancer progression. (a) A comparison of accumulated H3K4me3 profiles in a pair of matching metastasis and primary tumour cell lines. Shown are the number of ChIP-seq reads over $1 \mathrm{~kb}$ upstream and $1 \mathrm{~kb}$ downstream of transcription start site of each of 20,000 human genes in a matched pair of primary melanoma tumour (WM115, black, $x$ axis) and metastasis (WM266-4, blue, $y$ axis) cell lines derived from the same patient. The Pearson correlation coefficient is in the bottom right corner. (b,c) Density profiles of normalized reads from H3K4me3 ChIP-seq of primary melanoma tumour-derived cell line (WM115) or metastasis-derived cell line (WM266-4) at illustrative loci with either lower (b) or higher (c) levels of H3K4me3 in the metastasis-derived cell line. (d-g) Gene sets enriched (by GSEA) among loci with different levels of histone modifications. In each case, shown are the names of the enriched gene sets (rows) along with the normalized enrichment score, enrichment $P$-value and false discovery rates. Each entry in the heat map indicates the percent overlap in 'leading edge genes' (those that contribute to the enrichment) between two enriched gene sets. (d) Genes with lower H3K4me3 in metastasis cell line (WM266-4) relative to control primary tumour cell line (WM115). (e) Genes with higher H3K4me3 in metastasis cell line (WM266-4) relative to control primary tumour (WM115). (f) Genes with lower H3K4me3 in metastatic melanoma tumours from patients (an average of seven tumours samples) relative to control normal skin melanocytes (an average of three biological samples). (g) Genes with higher H3K4me3 in metastatic melanoma tumours from patients relative to control normal skin melanocytes. 
instructions. The process for sample preparation is outlined in Supplementary Fig. S1. Briefly, $45 \mu$ l of each ChIP DNA was added to a 96-well microtiter plate. Physical barcoding of all sample receptacles was employed at each step to ensure sample tracking integrity. Fragments were enzymatically end-repaired using an enzyme and buffer cocktail (Kapa Biosystems). Following end-repair, automated reaction clean-up was performed using a SPRI process with a ratio of 1.8 times beads to DNA (Ampure, Agencourt, Beckman Coulter). Samples were eluted in $30 \mu \mathrm{l}$ and added to a 50- $\mu$ l adaptor ligation reaction (ligase enzyme and buffer from Kapa Biosystems), which also contained $5 \mu \mathrm{l}$ of Ion Torrent compatible oligonucleotide adaptors (Integrated DNA Technologies). Adaptors were used at $8 \mu \mathrm{M}$ concentration (one-fifth of the standard amount) to minimize adaptor dimer with the low-input samples. A further $1.8 \times$ SPRI was performed after adaptor ligation. All automated fluid-handling steps were carried out on a Bravo Automated Liquid Handling Platform, with a 96LT Disposable Tip pipette head (Agilent Technologies, Santa Clara, CA). After adaptor ligation, $10 \mu \mathrm{l}$ of loading solution was added to each sample and each sample was size selected (280 base target size) using $2 \%$ gel cartridges (SAGE Pippin prep, Sage Science). A further SPRI $(2 \times$ Bead:DNA ratio) was performed after sizing and samples were eluted in $23 \mu \mathrm{l}$ volume. An amplification reaction was set up in a final volume of $50 \mu \mathrm{l}$ with the following cycling profile: $98^{\circ} \mathrm{C}$ for $45 \mathrm{~s} ; 72^{\circ} \mathrm{C}$ for $20 \mathrm{~min}$; followed by 12 cycles of $98^{\circ} \mathrm{C}$ for $15 \mathrm{~s}$, $63^{\circ} \mathrm{C}$ for $30 \mathrm{~s}$ and $72{ }^{\circ} \mathrm{C}$ for $30 \mathrm{~s}$; and finally, $72^{\circ} \mathrm{C}$ for $60 \mathrm{~s}$. Amplification enzymes and master mix were from Kapa Biosystems, and primers were those provided in a

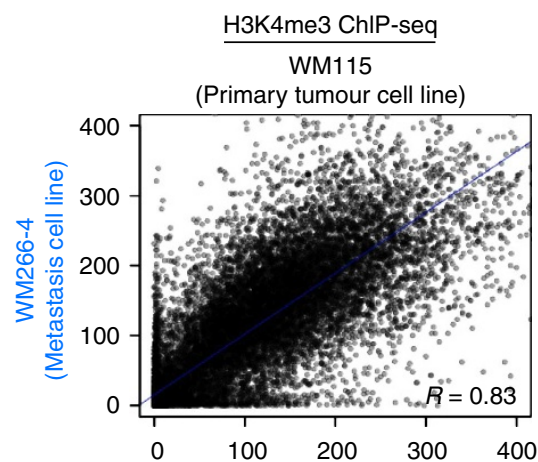

b

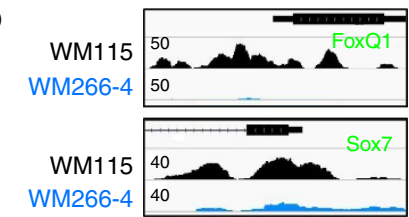

C

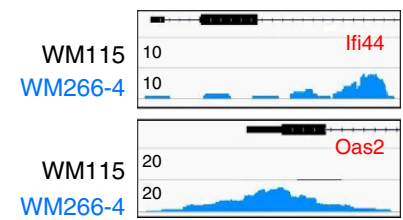

d

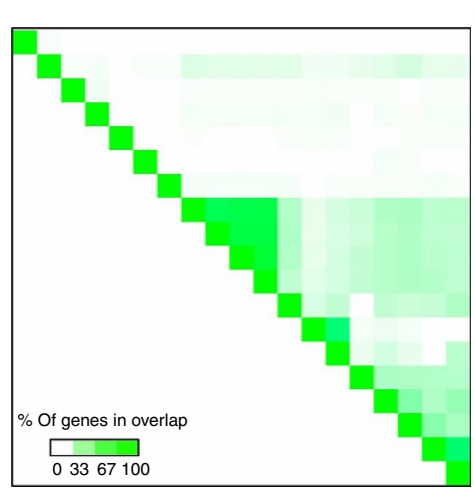

Name

WEBER_METHYLATED_LCP_IN_SPERM_UP

MARTENS_TRETINOIN_RESPONSE_UP

VALK_AML_WITH_11Q23_REARRANGED

LEE_TARGETS_OF_PTCH1_AND_SUFU_UP

ZHANG GATA6 TARGETS UP

MEISSNER_BRAIN_HCP_WITH_H3K4ME2

SCHLESINGER_H3K27ME3_IN_NORMAL_AND_METHYLATED_IN_CANCER $-1.8<0.0020 .02$

BENPORATH_ES_WITH_H3K27ME3 $\quad-2.1<0.0020 .00$ BENPORATH_SUZ12_TARGETS $\quad-2.1<0.002 \quad 0.00$ BENPORATH_EED_TARGETS

BENPORATH PRC2 TARGETS

MEISSNER BRAIN_HCP WITH H3K4ME3 AND_H3K27ME3

(1)

MIKKELSEN_NPC_HCP_WITH_H3K4ME3_AND_H3K27ME3

MEISSNER_BRAIN_HCP_WITH_H3K27ME3

MIKKELSEN_MCV6_HCP_WITH_H3K27ME3

MIKKELSEN_MEF_HCP_WITH_H3K27ME3

MIKKELSEN_NPC_HCP_WITH_H3K27ME3

MEISSNER_NPC_HCP_WITH_H3K4ME2_AND_H3K27ME3

Name

e

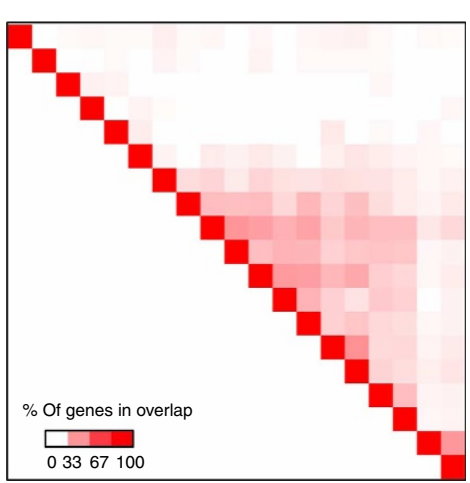

CHANG_CORE_SERUM_RESPONSE_DN NIKOLSKY_BREAST_CANCER_7Q21_Q22_AMPLICON ZIRN_TRETINOIN_RESPONSE_WT1_UP

CROONQUIST_NRAS_VS_STROMAL_STIMULATION_UP

VERRECCHIA_EARLY_RESPONSE_TO_TGFB 1

GILDEA_METASTASIS

FULCHER_INFLAMMATORY_RESPONSE_LECTIN_VS_LPS_DN

BOSCO_INTERFERON_INDUCED_ANTIVIRAL_MODULE

BROWNE_INTERFERON_RESPONSIVE_GENES

EINAV_INTERFERON_SIGNATURE_IN_CANCER

MOSERLE_IFNA_RESPONSE

ZHANG_INTERFERON_RESPONSE

DAUER_STAT3_TARGETS DN

SANA TNFING UP

SANA_RESPONSE_TO_IFNG_UP

DER_IFN_BETA_RESPONSE_UP

ZHU_CMV_8_HR_UP

MIKKELSEN_MEF_LCP_WITH_H3K4ME3

MIKKELSEN_MCV6_LCP_WITH_H3K4ME3

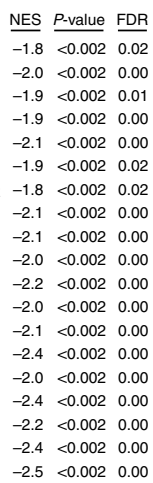

$\begin{array}{llll}-2.5 & <0.002 & 0.00\end{array}$

NES $P$-value FDR $\frac{\mathrm{ES}}{2.0}<0.001 \quad \frac{\mathrm{p}}{0.01}$ $2.1<0.001 \quad 0.00$ $2.0<0.001 \quad 0.00$ $2.0<0.001 \quad 0.00$ $2.1<0.001 \quad 0.00$ $2.1<0.001 \quad 0.00$ $2.2<0.001 \quad 0.00$ $2.0<0.001 \quad 0.01$ $2.5<0.001 \quad 0.00$ $2.3<0.001 \quad 0.00$ $\begin{array}{lll}2.1<0.001 & 0.00\end{array}$ $2.4<0.001 \quad 0.00$ $2.2<0.001 \quad 0.00$ $2.1<0.001 \quad 0.00$ $2.1<0.001 \quad 0.00$ $2.2<0.001 \quad 0.00$ $2.0<0.001 \quad 0.01$ $\begin{array}{lll}2.0 & <0.001 & 0.01 \\ 2.0 & <0.001 & 0.01\end{array}$ $2.0<0.001 \quad 0.01$ $2.0<0.001 \quad 0.00$ $2.0<0.001 \quad 0.01$ f

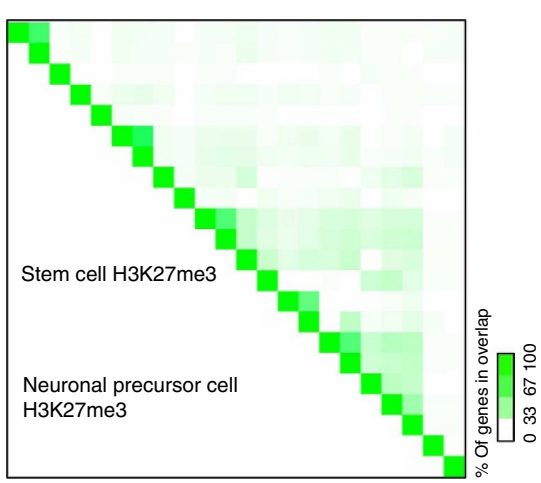

g

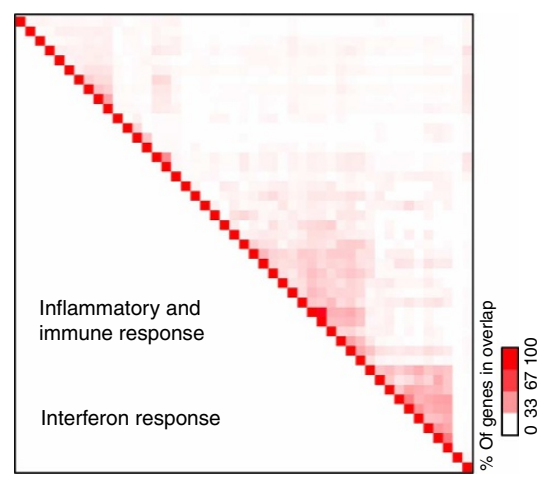


the Ion Torrent template preparation kit. A SPRI clean-up with a $1.5 \times$ Bead:DNA ratio was performed after amplification and final libraries were eluted in $25 \mu \mathrm{l}$ volume. Libraries were quantified and checked for size on an Agilent Bioanalyzer (Agilent Technologies).

Template preparation was conducted using the Ion PGM 200 Xpress Template Kit, following the Ion PGM 200 Xpress Template Kit protocol (version 3). Libraries were diluted and $18 \mu \mathrm{l}$ of the $1.55 \times 10^{7}$ molecules per $\mu \mathrm{l}$ dilution was added to an aqueous master mix containing polymerase and ion sphere particles (ISPs) according to the manufacturer's specified proportions. Emulsions were created using an IKA Ultra-Turrax Tube Drive (IKA, Wilmington, NC). After emulsion PCR, DNA-positive ISPs were recovered and enriched according to standard protocols. A sequencing primer was annealed to DNA-positive ISPs and the sequencing polymerase was bound before loading of ISPs into Ion 316 sequencing chips. Sequencing of the samples was conducted according to the Ion PGM 200 Sequencing Kit Protocol (version 6). One or more 316 sequencing chips were loaded and run on an Ion Personal Genome Machine for each sample. Each run was programmed to include 520 nucleotide flows to deliver 200 base read lengths on average. Libraries were sequenced on Ion Torrent Personal Genome Machine. Base calling and alignment were performed by the Torrent Suite 2.0.1 software.

Illumina library production and sequencing. ChIP-seq library for Illumina sequencing were prepared using a previously published protocol ${ }^{28}$. Briefly, enzymes from New England Biolabs were used for the following library construction processes, DNA end-repair, A-base addition and adaptor ligation, and Pfu Ultra II fusion enzyme (Agilent Technologies) was used for the enrichment step. Illumina ChIP libraries were barcoded and pooled as previously described ${ }^{28}$.

ChIP-seq analysis. Reads were aligned to the reference mouse genome (mm9) or human genome (hg19) using the BWA aligner version 0.5.9. Sequencing metrics were extracted using the GATK tools ${ }^{29}$ to traverse the genome and qualify mapped bases as well aligned-when the reads had mapping quality greater than Q20 (phred scaled) and high quality - when the reads had mapping quality greater than Q20 and the base had base quality greater than Q20. Error rates were measured in every 100 bases. Mismatches were counted for every base that mismatched the reference sequence in the alignment. Insertions and deletions were counted as events (not by the number of bases in the events). The rate is the number of insertion or deletion events found in every 100 bases. Read length was calculated over all reads (including unmapped). Unmapped reads are all the reads that did not find a likely mapping in the mouse reference genome $\mathrm{mm} 9$ (this does not include mapping quality 0 reads). ChIP-seq peak calling was performed using the contiguous segmentation algorithm as part of the Scripture package ${ }^{28}$ (http:// www.broadinstitute.org/software/scripture/). Pearson's correlation coefficients in Fig. 1 were calculated by performing Pearson's correlation analysis of pairwise comparison of ChIP-seq peak enrichment scores $(\log 2)$ over a 500-base sliding window.

To simulate for longer reads, we extended the 40-base Illumina reads to 180 bases by setting the extFactor flag to 140 in igvtools (http://www.broadinstitute.org/ software/igv/igvtools_commandline). To simulate for shorter Ion Torrent reads, we selected the first 40 bases from the 180 bases of Ion Torrent reads. The sequencing data can be downloaded from the NCBI GEO database with the following accession number GSE49477.

GSEA analysis. GSEA v2.2 was used to test for enrichment of each of the 3,398 gene sets in the chemical and genetic perturbation collection of the Molecular Signature Database (MSigDB v3.1). Reads were first normalized based on the total number of reads for each sample. For each of the 20,000 NCBI human Refseg genes, the accumulated H3K4me3 ChIP-seq reads over $1 \mathrm{~kb}$ upstream and $1 \mathrm{~kb}$ downstream of transcription start site was calculated to present the H3K4me3 ChIP-seq signal for each gene. The residuals of the natural logarithms of the accumulated reads calculated from the linear model for each gene were used as the ranked list input for the GSEAPreranked function. Three biological repeats of H3K4me3 ChIP-seq Illumina sequencing data from normal skin melanocytes were downloaded from NCBI GEO database $\left(\right.$ GSE16368) ${ }^{30}$.

\section{References}

1. Rothberg, J. M. et al. An integrated semiconductor device enabling non-optical genome sequencing. Nature 475, 348-352 (2011).

2. Ferain, I., Colinge, C. A. \& Colinge, J. P. Multigate transistors as the future of classical metal-oxide-semiconductor field-effect transistors. Nature 479, 310-316 (2011).

3. Glenn, T. C. Field guide to next-generation DNA sequencers. Mol. Ecol. Resour. 11, 759-769 (2011)

4. Merriman, B. \& Rothberg, J. M. Progress in ion torrent semiconductor chip based sequencing. Electrophoresis 33, 3397-3417 (2012).

5. Vogel, U. et al. Ion torrent personal genome machine sequencing for genomic typing of Neisseria meningitidis for rapid determination of multiple layers of typing information. J. Clin. Microbiol. 50, 1889-1894 (2012).
6. Elliott, A. M., Radecki, J., Moghis, B., Li, X. \& Kammesheidt, A. Rapid detection of the ACMG/ACOG-recommended 23 CFTR disease-causing mutations using ion torrent semiconductor sequencing. J. Biomol. Tech. 23, 24-30 (2012).

7. Ernst, J. et al. Mapping and analysis of chromatin state dynamics in nine human cell types. Nature 473, 43-49 (2011).

8. Gerstein, M. B. et al. Architecture of the human regulatory network derived from ENCODE data. Nature 489, 91-100 (2012).

9. Ram, O. et al. Combinatorial patterning of chromatin regulators uncovered by genome-wide location analysis in human cells. Cell 147, 1628-1639 (2011).

10. Adli, M. \& Bernstein, B. E. Whole-genome chromatin profiling from limited numbers of cells using nano-ChIP-seq. Nat. Protoc. 6, 1656-1668 (2011).

11. Adli, M., Zhu, J. \& Bernstein, B. E. Genome-wide chromatin maps derived from limited numbers of hematopoietic progenitors. Nat. Methods 7, 615-618 (2010).

12. Lennon, N. J. et al. A scalable, fully automated process for construction of sequence-ready barcoded libraries for 454. Genome Biol. 11, R15 (2010).

13. Quail, M. A. et al. Optimal enzymes for amplifying sequencing libraries. Nat. Methods 9, 10-11 (2012)

14. Landt, S. G. et al. ChIP-seq guidelines and practices of the ENCODE and modENCODE consortia. Genome Res. 22, 1813-1831 (2012).

15. ENCODE Project Consortium. A user's guide to the encyclopedia of DNA elements (ENCODE). PLoS Biol. 9, e1001046 (2011).

16. Subramanian, A. et al. Gene set enrichment analysis: a knowledge-based approach for interpreting genome-wide expression profiles. Proc. Natl Acad. Sci. USA 102, 15545-15550 (2005).

17. Lee, T. I. et al. Control of developmental regulators by Polycomb in human embryonic stem cells. Cell 125, 301-313 (2006).

18. Bernstein, B. E. et al. A bivalent chromatin structure marks key developmental genes in embryonic stem cells. Cell 125, 315-326 (2006).

19. Ben-Porath, I. et al. An embryonic stem cell-like gene expression signature in poorly differentiated aggressive human tumors. Nat. Genet. 40, 499-507 (2008).

20. Kim, J. et al. A Myc network accounts for similarities between embryonic stem and cancer cell transcription programs. Cell 143, 313-324 (2010).

21. Chi, P., Allis, C. D. \& Wang, G. G. Covalent histone modifications--miswritten, misinterpreted and mis-erased in human cancers. Nat. Rev. Cancer 10, 457-469 (2010).

22. Min, J. et al. An oncogene-tumor suppressor cascade drives metastatic prostate cancer by coordinately activating Ras and nuclear factor-kappaB. Nat. Med. 16, 286-294 (2010).

23. Tsang, D. P. \& Cheng, A. S. Epigenetic regulation of signaling pathways in cancer: role of the histone methyltransferase EZH2. J. Gastroenterol. Hepatol. 26, 19-27 (2011).

24. Yu, J. et al. A polycomb repression signature in metastatic prostate cancer predicts cancer outcome. Cancer Res. 67, 10657-10663 (2007).

25. Ben-Neriah, Y. \& Karin, M. Inflammation meets cancer, with NF-kappaB as the matchmaker. Nat. Immunol. 12, 715-723 (2011).

26. Coussens, L. M. \& Werb, Z. Inflammation and cancer. Nature 420, 860-867 (2002).

27. Mantovani, A., Allavena, P., Sica, A. \& Balkwill, F. Cancer-related inflammation. Nature 454, 436-444 (2008).

28. Garber, M. et al. A high-throughput chromatin immunoprecipitation approach reveals principles of dynamic gene regulation in mammals. Mol. Cell 47, 810-822 (2012).

29. DePristo, M. A. et al. A framework for variation discovery and genotyping using next-generation DNA sequencing data. Nat. Genet. 43, 491-498 (2011).

30. Bernstein, B. E. et al. The NIH Roadmap Epigenomics Mapping Consortium. Nat. Biotechnol. 28, 1045-1048 (2010).

\section{Acknowledgements}

We thank R. Satija and S. Schwartz for helpful discussions. We thank J. Bochicchio for project management, A. Wysoker for the development of the data transfer pipeline and the Broad's Genomics Platform for sequencing. We also thank T. L. Calderone for preparing and sending the melanoma tumours. We thank L. Gaffney for production of the graphical abstract. This work was supported by the Human Frontiers Science Program Career Development Award (I.A.), the ISF Bikura Institutional Research Grant Program (I.A.), ERC starting Grant 309788 (I.A.), NHGRI (1P50HG006193-01 to I.A. and A.R.), NIH Pioneer Award (DP1OD003958-01 to A.R.), Klarman Cell Observatory (A.R.), NCI (R01 CA93947 to L.C.), NCI (U01 CA1411508 to L.C.), NHGRI (U54HG003067 to C.R.), NIH (P50 CA093459 to J.G.) and NIH Ruth L. Kirschstein National Research Service awards for individual Postdoctoral Fellowships (C.S.C.).

\section{Author contributions}

C.S.C., A.R. and I.A. designed the study. C.S.C. performed the experiments and conducted data analysis. N.L., A.H., D.R., S.A., A.R. and A.T. developed and conducted the Ion Torrent ChIP-seq library construction experiments. C.S.C., A.R. and I.A. wrote the manuscript, with help from C.N., C.R. and N.H. A.R. guided data analysis. I.A. guided experimental designs. L.C. provided expertise and guidance in melanoma biology and 
analysis. K.R. provided fixed melanoma cell pellets from tumour-derived cell lines and assisted in design of melanoma-related experiments. M.G. provided analysis expertise and aided in the analysis of the data. M.O.C. carried out error rate analysis and helped with writing. R.R. carried out mouse dendritic cell culturing and fixing the cell pellets. J.E.G. and L.C. collected and provided tumour samples from melanoma patients. C.N., C.R. and N.H. helped editing the manuscript. All authors edited the manuscript.

\section{Additional information}

Accession Codes: Sequencing data have been uploaded to the NCBI GEO database under Accession Number GSE49477.
Supplementary Information accompanies this paper at http://www.nature.com/ naturecommunications

Competing financial interests: The authors declare no competing financial interests.

Reprints and permission information is available online at http://npg.nature.com/ reprintsandpermissions/

How to cite this article: Cheng, C. S. et al. Semiconductor-based DNA sequencing of histone modification states. Nat. Commun. 4:2672 doi: 10.1038/ncomms3672 (2013). 